\title{
A New Method for Power Signal Harmonic Analysis
}

\author{
Jun-Zhe Yang, Member, IEEE, Chi-Shan Yu, and Chih-Wen Liu, Senior Member, IEEE
}

\begin{abstract}
With the increasing use of nonlinear loads in power systems, the harmonic pollution becomes more and more serious. It is well known that fast Fourier transform (FFT) is a powerful tool for power signal harmonic analysis, but leakage effect, picket fence effect, and aliasing effect make FFT suffer from specific restrictions. In this paper, we proposed a new method for power signal harmonic analysis. The major components of this method are a frequency and phasor estimating algorithm, a finite-impulse-response comb filter, and a correction factor. We also combine other methods to enhance our performance, such as discrete Fourier transform and least square error (LSE) method. To verify this method, we provided the comparisons of this method with FFT.
\end{abstract}

Index Terms-Fast Fourier transform (FFT), finite-impulse-response (FIR) comb filter, harmonic analysis.

\section{INTRODUCTION}

W ITH the progress of industry, power-electronic equipment is widely used in power systems, but the nonlinear characteristics of this equipment have also produced serious harmonic pollution. In addition, many ill effects (i.e., worse power quality for end users, more loss in transmission lines, overheating of machines, and malfunction of relays and breakers) are due to harmonic pollution. It goes without saying that harmonic analysis is a very important subject in power systems.

About harmonic analysis, several algorithms have been proposed [1]-[5], and fast Fourier transform (FFT) is the most widely used computation algorithm for harmonic analysis [5]-[7]. However, leakage effect, picket-fence effect, and aliasing effect make FFT suffer from specific restrictions. Therefore, some methods [7]-[13] have also been provided to improve these drawbacks.

The harmonic analysis method we proposed in this paper is composed of three different parts: an accurate frequency and phasor estimation algorithm, an FIR comb filter to filter out specific signal components, and a correction factor to eliminate the side effect of the FIR comb filter.

The organization of this paper is as follows: we describe an algorithm for estimating the frequency and phasor in Section II. Next, in Section III, an FIR comb filter is derived from the results of Section II. Then, we successfully eliminate the side effect of the FIR comb filter in Section IV. The method we use

Manuscript received November 20, 2003. Paper no. TPWRD-00579-2003.

J.-Z. Yang is with the Department of Electrical Engineering, I-Shou University, Kaoshiung 840, Taiwan, R.O.C. (e-mail: jzyang@ @isu.edu.tw).

C.-S. Yu is with the Department of Electrical Engineering, Chung-Cheng Institute of Technology, National Defense University, Taoyuan 335, Taiwan, R.O.C. (e-mail: chsyu@ @cit.edu.tw).

C.-W. Liu is with the Department of Electrical Engineering, National Taiwan University, Taipei 106, Taiwan, R.O.C. (e-mail: cwliu@cc.ee.ntu.edu.tw).

Digital Object Identifier 10.1109/TPWRD.2004.834311 to conduct harmonic analysis is proposed in Section V. In Section VI, simulation results are presented. Finally, we give conclusions in Section VII.

\section{Frequency AND Phasor Estimation Algorithm}

This section presents the algorithm for estimating frequency and phasor from a power signal. Suppose the signal $x(t)$ is assumed to be of the form

$$
x(t)=X \cos (2 \pi f t+\phi)
$$

where $X$ is the amplitude of the signal; $f$ is the frequency of the signal; and $\phi$ is the phase angle of the signal. Suppose that $x(t)$ is sampled with a fixed time interval $(\Delta t)$ to produce the sampled set $\{x(k)\}$

$$
x(k)=X \cos (2 \pi f k \Delta t+\phi) .
$$

Since we know that

$$
\cos \theta=\frac{e^{j \theta}+e^{-j \theta}}{2}
$$

Then, $x(k)$ can be expressed as

$$
x(k)=\frac{X}{2} e^{j \phi} e^{j 2 \pi f k \Delta t}+\frac{X}{2} e^{-j \phi} e^{-j 2 \pi f k \Delta t} .
$$

For further explorations, we use the following definitions:

$$
\begin{aligned}
a & =e^{j 2 \pi f \Delta t} \\
A & =\frac{X}{2} e^{j \phi} \\
z & =\operatorname{Re}(a) .
\end{aligned}
$$

Then, (4) can be expressed as

$$
x(k)=A a^{k}+A^{*} a^{-k} .
$$

Therefore, we can find the following relations between $x(k)$ and $x(k+1)$.

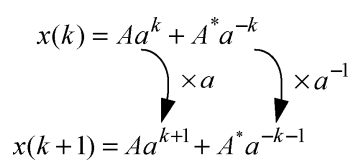

After some algebraic manipulations, we find

$$
z=\frac{x(k)+x(k+2)}{2 x(k+1)} .
$$

According to the definitions given above, we can compute the parameters of signal by the following equations:

$$
\begin{aligned}
& f=\frac{\cos ^{-1}(z)}{2 \pi \Delta t} \\
& A=\frac{x(k+1) a-x(k)}{\left(a^{2}-1\right)}
\end{aligned}
$$




$$
\begin{aligned}
X & =2|A| \\
\phi & =\operatorname{angle}(A) .
\end{aligned}
$$

From the above equations, we find that this algorithm is easy to implement and not affected by frequency deviation. In addition, we find an important relation from (9)

$$
x(k)-2 z x(k+1)+x(k+2)=0 .
$$

Equation (14) reveals an important massage: if we can find the correct value of "z," then we can filter out this component of the frequency from signal. This idea can be expressed by the following.

$$
\begin{aligned}
& x(k) \longrightarrow \text { FIR filter } \longrightarrow y(k) \\
& x(k)-2 z x(k+1)+x(k+2)=y(k+2)
\end{aligned}
$$

\section{FIR COMB FILTER}

According to the results of Section II, we continue to develop advanced features. We want to transfer the algorithm in Section II into an FIR comb filter. Consider the waveform of a power signal as follows:

$$
x(t)=\sum_{i=1}^{\mathrm{m}} X_{i} \cos \left(2 \pi f_{i} t+\phi_{i}\right) .
$$

Suppose that $x(t)$ is sampled with a fixed time interval $(\Delta t)$ to produce the sampled set $\{x(k)\}$

$$
x(k)=\sum_{i=1}^{m} X_{i} \cos \left(2 \pi f_{i} k \Delta t+\phi_{i}\right) \quad \mathrm{k}=0,1,2, \ldots
$$

Then, we define

$$
\begin{aligned}
a_{i} & =e^{j 2 \pi f_{i} \Delta t} \\
A_{i} & =\frac{X_{i}}{2} e^{j \phi_{i}} \\
z_{i} & =\operatorname{Re}\left(a_{i}\right) .
\end{aligned}
$$

After some algebraic manipulations, we can find out the following results:

$$
\begin{aligned}
& \sum_{n=0}^{2 m} C(n) x(k+n)=0 \\
& C=\left\{\left\{1,-2 z_{1}, 1\right\} *\left\{1,-2 z_{2}, 1\right\} * \ldots *\left\{1,-2 z_{m}, 1\right\}\right\}
\end{aligned}
$$

where $*$ means convolution operator.

According to (20), we can arbitrarily filter the harmonic components in the signal if we obtain the correct values of $C(n)$. Since harmonics are multiple of the fundamental frequency, $f_{1}$ is the key to obtain the correct values of $C(n)$.

\section{WINDOW CORRECTION FACTOR}

Although the proposed FIR comb filter can filter out the desired frequency components from the signal, it still has some side effects. It changes the amplitude and phase of the remaining components of the signal. To eliminate the side effect of the proposed filter, we provide window correction factor (WCF). Suppose a sampled set $\{x(k)\}$ becomes a filtered set $\{\widetilde{x}(k)\}$ by an FIR window filter $W$

$$
\widetilde{x}(k)=\sum_{n=0}^{M-1} W(n) x(k+n) .
$$

We rearrange (22) to obtain

$$
\widetilde{x}(k)=A a^{k} \sum_{n=0}^{M-1} W(n) a^{n}+A^{*} a^{-k} \sum_{n=0}^{M-1} W(n) a^{n} .
$$

Then, we define

$$
\widetilde{A}=A \sum_{n=0}^{M-1} W(n) a^{n} .
$$

Therefore, (23) can be expressed as follows:

$$
\widetilde{x}(k)=\widetilde{A} a^{k}+\widetilde{A}^{*} a^{-k} .
$$

From (25), we can find the relations between $\widetilde{x}(k)$ and $\widetilde{x}(k+1)$ are the same as the relations between $x(k)$ and $x(k+1)$.

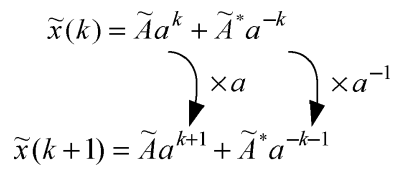

After some algebraic manipulations, we obtain

$$
z=\frac{\widetilde{x}(k)+\widetilde{x}(k+2)}{2 \widetilde{x}(k+1)} .
$$

According to the definitions given above, we can compute the parameters of the signal by the following equations:

$$
\begin{aligned}
& f=\frac{\cos ^{-1}(z)}{2 \pi \Delta t} \\
& A=\frac{\widetilde{x}(k+1) a-\widetilde{x}(k)}{\left(a^{2}-1\right) \times W C F} .
\end{aligned}
$$

We define WCF as follows:

$$
\mathrm{WCF}=\sum_{n=0}^{M-1} W(n) a^{n} .
$$

Moreover, according to the definition of $W$, WCF is suitable for any finite sequence of the digital filter. Therefore, not only is the filter we proposed suitable, but so are window filters such as Hamming, Hanning, and Blackman window. Even discrete Fourier transform (DFT), a finite complex sequence, is also included.

\section{Proposed Harmonic Analysis Method}

The way we conduct harmonic analysis is different from FFT. Simply speaking, we compute each harmonic separately. Meanwhile, to prevent the influence of other harmonics, we have to filter them out. Moreover, if we obtain the fundamental frequency, then we can obtain the frequency of each harmonic. 


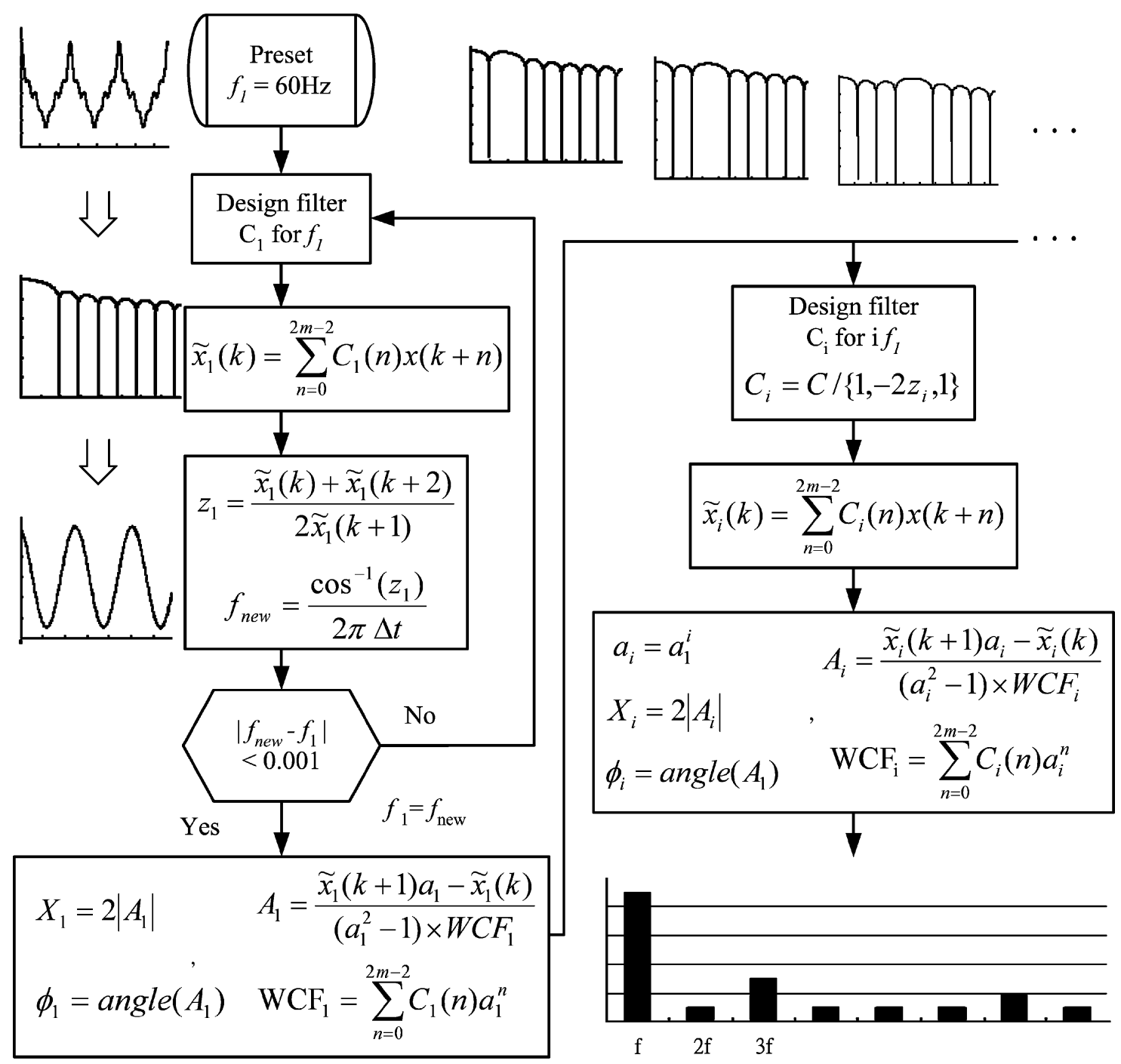

Fig. 1. Process of harmonic analysis.

Therefore, knowing how to estimate fundamental frequency is the key point. The procedure of our harmonic analysis is discussed: First, assume fundamental frequency is $60 \mathrm{~Hz}$, then use (21) to create a comb filter, which filters out every harmonic. After filtering, we find the new fundamental frequency. Then, we redesign the comb filter for the new fundamental frequency and repeat these steps until the fundamental frequency converges. Next, produce the combs filter to filter out every harmonic except the $i$ th harmonic and compute the phasor of the $i$ th harmonic. The process is shown in Fig. 1.

In Fig. 1

$$
C_{1}=\left\{\left\{1,-2 z_{2}, 1\right\} * \ldots *\left\{1,-2 z_{m}, 1\right\}\right\}
$$

To reduce the computation time, we utilize deconvolution to get $C_{i}$. After getting $f_{1}$, we also get $C_{1}$ at the same time. Then, we can get $C$ by convolution

$$
C=C_{1} *\left\{1,-2 z_{1}, 1\right\}
$$

Next, we get $C_{i}$ by deconvolution (/)

$$
C_{i}=\frac{C}{\left\{1,-2 z_{i}, 1\right\}} .
$$

We can get the $\widetilde{x}_{i}(k)$ by the following equation:

$$
\widetilde{x}_{i}(k)=\sum_{n=0}^{2 m-2} C_{i}(n) x(k+n) .
$$

Next, we only compute the phasor of each harmonic by

$$
A_{i}=\frac{\widetilde{x}_{i}(k+1) a_{i}-\widetilde{x}_{i}(k)}{\left(a_{i}^{2}-1\right) \times W C F_{i}} .
$$

By using this method for power signal harmonic analysis, we will not seriously suffer the leakage effect, picket fence effect, and aliasing effect. Moreover, the sampling frequency is not necessary to be $2 \mathrm{n}$ multiples of the fundamental frequency. Although this method is not as fast as FFT, it is suitable for both online and offline applications. Additionally, it is obvious that 
this method is more efficient in parallel than in serial computation.

Since the accuracy of the fundamental frequency is the key point, we provide the LSE method to enhance the performance. We rewrite (26) as follows:

$$
z_{1}\left[\begin{array}{c}
2 \widetilde{x}(k+1) \\
\vdots \\
2 \widetilde{x}(k+L+1)
\end{array}\right]=\left[\begin{array}{c}
\widetilde{x}(k)+\widetilde{x}(k+2) \\
\vdots \\
\widetilde{x}(k+L)+\widetilde{x}(k+L+2)
\end{array}\right] .
$$

Then, $z_{1}$ can be obtained by

$$
z_{1}=\left(A^{\prime} A\right)^{-1} A^{\prime} B
$$

where $\mathrm{L}$ is the window size of the LSE method and

$$
A=\left[\begin{array}{c}
2 \widetilde{x}(k+1) \\
\vdots \\
2 \widetilde{x}(k+L+1)
\end{array}\right], \quad B=\left[\begin{array}{c}
\widetilde{x}(k)+\widetilde{x}(k+2) \\
\vdots \\
\widetilde{x}(k+L)+\widetilde{x}(k+L+2)
\end{array}\right] \text {. }
$$

\section{Simulation RESUlts}

Here, we present two simulation results comparing the method we proposed with the FFT. For convenience, the method we proposed is called a combined method (CM). In the first case, the fundamental frequency is $59.5 \mathrm{~Hz}$, and the harmonic contents are shown in Table I. We add $0.5 \%$ white noise into the signal. The sampling frequency is $60 \times 128=7680 \mathrm{~Hz}$ because of the restriction of FFT. The computation results are also shown in Table I.

In this case, we use Matlab built-in function "FFT" to compute the results of FFT, and the window size of FFT is 128 samples. There are two different window sizes of CM: one is for computing the fundamental frequency and another is for computing harmonic contents. The window size of the computing fundamental frequency is 265 samples (127 for the comb filter, 128 for DFT, and ten for LSE). The window size of computing harmonic contents is changing with the order $i$ of the harmonic (127 for comb filter, round (128/i) for DFT). From Table I, we can compare the performances of both methods. CM is better than FFT; this is because CM is not affected by frequency deviation. Moreover, if we want the performance of CM to be much better than FFT, we can add smoothing window like Hanning or Blackman window to filter out noise. In addition, the side effect of the smoothing window also can be eliminated by WCF.

In the second case, we discuss the aliasing effect. Assume the fundamental frequency is $60.5 \mathrm{~Hz}$ and the sampling frequency is $60 \times 16=960 \mathrm{~Hz}$. The harmonic contents and simulation results are shown in Table II. Since the fundamental frequency is $60.5 \mathrm{~Hz}$, the frequency of the eighth harmonic is $484 \mathrm{~Hz}$, which is above half of the sampling frequency. According to the Nyquist sampling theorem, this part of the signal cannot be recovered from samples. However, the influences of these harmonics still exist. The computation results of FFT are affected, which is called the aliasing effect. From Table II, we can find that CM gets the correct harmonic contents under no-noise condition, but it does not mean CM is out of the Nyquist sampling theorem. Actually from (14), we can find

$$
z_{8}=\cos (2 \pi 484 \Delta t)=\cos (2 \pi 476 \Delta t) .
$$

TABLE I

Simulation Signal AND ReSults (F1 $=59.5 \mathrm{~Hz}$ With 0.5\% NoISE)

\begin{tabular}{l|r|c|c|c|r|c|c}
\hline H & Amp. & FFT & CM & H & Amp. & FFT & CM \\
\hline 1 & 1 & 0.9931 & 0.9997 & 33 & 0 & 0.0031 & 0.0003 \\
\hline 2 & 0.07 & 0.0737 & 0.0702 & 34 & 0 & 0.0034 & 0.0004 \\
\hline 3 & 0.05 & 0.0526 & 0.0503 & 35 & 0 & 0.0033 & 0.0002 \\
\hline 4 & 0 & 0.0016 & 0.0005 & 36 & 0 & 0.0032 & 0.0003 \\
\hline 5 & 0.04 & 0.0411 & 0.0399 & 37 & 0 & 0.0033 & 0.0001 \\
\hline 6 & 0 & 0.0018 & 0.0003 & 38 & 0 & 0.0041 & 0.0005 \\
\hline 7 & 0.03 & 0.0320 & 0.0298 & 39 & 0 & 0.0040 & 0.0001 \\
\hline 8 & 0 & 0.0036 & 0.0001 & 40 & 0 & 0.0044 & 0.0001 \\
\hline 9 & 0 & 0.0028 & 0.0003 & 41 & 0 & 0.0048 & 0.0002 \\
\hline 10 & 0 & 0.0023 & 0.0005 & 42 & 0 & 0.0051 & 0.0004 \\
\hline 11 & 0 & 0.0023 & 0.0004 & 43 & 0 & 0.0060 & 0.0004 \\
\hline 12 & 0 & 0.0018 & 0.0002 & 44 & 0 & 0.0077 & 0.0004 \\
\hline 13 & 0 & 0.0017 & 0.0001 & 45 & 0 & 0.0108 & 0.0002 \\
\hline 14 & 0 & 0.0017 & 0.0000 & 46 & 0 & 0.0217 & 0.0003 \\
\hline 15 & 0 & 0.0018 & 0.0002 & 47 & 0.03 & 0.0130 & 0.0298 \\
\hline 16 & 0 & 0.0023 & 0.0005 & 48 & 0 & 0.0209 & 0.0001 \\
\hline 17 & 0 & 0.0019 & 0.0003 & 49 & 0.05 & 0.0396 & 0.0499 \\
\hline 18 & 0 & 0.0020 & 0.0001 & 50 & 0 & 0.0123 & 0.0002 \\
\hline 19 & 0 & 0.0020 & 0.0002 & 51 & 0 & 0.0075 & 0.0003 \\
\hline 20 & 0 & 0.0028 & 0.0003 & 52 & 0 & 0.0056 & 0.0003 \\
\hline 21 & 0 & 0.0036 & 0.0002 & 53 & 0 & 0.0042 & 0.0001 \\
\hline 22 & 0 & 0.0066 & 0.0002 & 54 & 0 & 0.0038 & 0.0001 \\
\hline 23 & 0.02 & 0.0154 & 0.0197 & 55 & 0 & 0.0039 & 0.0002 \\
\hline 24 & 0 & 0.0065 & 0.0002 & 56 & 0 & 0.0044 & 0.0001 \\
\hline 25 & 0.04 & 0.0394 & 0.0399 & 57 & 0 & 0.0060 & 0.0002 \\
\hline 26 & 0 & 0.0087 & 0.0003 & 58 & 0 & 0.0150 & 0.0003 \\
\hline 27 & 0 & 0.0056 & 0.0002 & 59 & 0.02 & 0.0106 & 0.0203 \\
\hline 28 & 0 & 0.0044 & 0.0001 & 60 & 0 & 0.0055 & 0.0001 \\
\hline 29 & 0 & 0.0040 & 0.0002 & 61 & 0.01 & 0.0067 & 0.0098 \\
\hline 30 & 0 & 0.0036 & 0.0000 & 62 & 0 & 0.0034 & 0.0003 \\
\hline 31 & 0 & 0.0035 & 0.0002 & 63 & 0 & 0.0089 & 0.0003 \\
\hline 32 & 0 & 0.0033 & 0.0001 & 64 & 0.01 & 0.0017 & 0.0102 \\
\hline & & & & & & & \\
\hline
\end{tabular}

TABLE II

Simulation Signal AND Results $(\mathrm{F} 1=60.5 \mathrm{~Hz})$

\begin{tabular}{l|r|c|c|c|c}
\hline \multicolumn{2}{c|}{$\mathrm{f}_{1}=60.5 \mathrm{~Hz}$} & \multicolumn{2}{c|}{ No Noise } & \multicolumn{2}{c}{$0.5 \%$ Noise } \\
\hline $\mathrm{H}$ & Amp. & FFT & CM & FFT & CM \\
\hline 1 & 1 & 1.0071 & 1.0000 & 1.0061 & 0.9998 \\
\hline 2 & 0.07 & 0.0668 & 0.0700 & 0.0673 & 0.0704 \\
\hline 3 & 0.05 & 0.0481 & 0.0500 & 0.0481 & 0.0500 \\
\hline 4 & 0 & 0.0009 & 0.0000 & 0.0011 & 0.0006 \\
\hline 5 & 0.04 & 0.0388 & 0.0400 & 0.0384 & 0.0402 \\
\hline 6 & 0 & 0.0019 & 0.0000 & 0.0028 & 0.0003 \\
\hline 7 & 0.03 & 0.0271 & 0.0300 & 0.0264 & 0.0293 \\
\hline 8 & 0.01 & 0.0141 & 0.0100 & 0.0152 & 0.0102 \\
\hline
\end{tabular}

The signal components over half of the sampling frequency are reflected back, and we cannot tell what these are. However, using the method we proposed will allow us to avoid an aliasing effect when the fundamental frequency is not exactly at $60 \mathrm{~Hz}$. In this case, we add a Blackman window to enhance the preference while we add the noise into the signal.

\section{CONCLUSION}

In this paper, we provide a new measurement method for harmonic analysis. This method is easy to implement and very flexible. Users can change the window (smoothing window, DFT, or LSE) and window size to get better performance. Meanwhile, it does not seriously suffer the drawbacks like leakage effect, picket-fence effect, and sampling frequency 
have to be $2^{\mathrm{n}}$ multiple of the fundamental frequency. It is also more able to deal with the aliasing effect. This method really meets the need of offline applications. Furthermore, if we can implement this method in parallel computation, it should meet the need of online applications and be more practical.

\section{REFERENCES}

[1] A. Mehorai and B. Porat, "Adaptive comb filtering for harmonic signal enhancement," IEEE Trans. Acoust. Speech Signal Processing, vol. ASSP-34, no. 5, pp. 1124-1138, Oct. 1986.

[2] G. Takata, J. Tahara, M. Michihira, A. Tsuyoshi, K. Amako, H. Omori, and K. Yasui, "The time-frequency analysis of the harmonics with wavelet transform for the power electronics systems," in Proc. Power Conversion Conf., vol. 2, Apr. 2002, pp. 733-737.

[3] Y. Z. Liu and S. Chen, "A wavelet based model for on-line tracking of power system harmonics using Kalman filtering," in Proc. IEEE Power Engineering Society Summer Meet., vol. 2, Jul. 2001, pp. 1237-1242.

[4] H. Xue and R. Yang, "A novel algorithm for harmonic measurement in power system," in Proc. Int. Conf. PowerCon, vol. 1, Oct. 2002, pp. 438-442.

[5] M. Meunier and F. Brouaye, "Fourier transform, wavelets, Prony analysis: tools for harmonics and quality of power," in Proc. 8th Int. Conf. Harmonics Quality Power, vol. 1, Oct. 1998, pp. 71-76.

[6] A. A. Girgis, The Fast Fourier Transform and Its Applications. Upper Saddle River, NJ: Prentice-Hall, 1990.

[7] A. N. Mortensen and G. L. Johnson, "A power system digital harmonic analyzer," IEEE Trans. Instrum. Meas., vol. 37, no. 4, pp. 537-540, Dec. 1988.

[8] T. P. Tsao, R. C. Wu, and C. C. Ning, "The optimization of spectral analysis for signal harmonics," IEEE Trans. Power Del., vol. 16, pp. 149-153, Apr. 2001.

[9] F. Zhang, Z. Geng, and W. Yuan, "The algorithm of interpolating windowed FFT for harmonic analysis of electric power system," IEEE Trans. Power Del., vol. 16, no. 2, pp. 160-164, Apr. 2001.

[10] W. Pan, Y. S. Qian, and E. Zhou, "An improved interpolated FFT algorithm and its application in power harmonics measurement," in Proc. IEEE Int. Conf. Industrial Technology, Dec. 1994, pp. 273-277.

[11] F. Zhang, Z. Geng, and W. Yuan, "The algorithm of interpolating windowed FFT for harmonic analysis of electric power system," IEEE Trans. Power Del., vol. 16, no. 2, pp. 160-164, Apr. 2001.

[12] H. C. Lin and C. S. Lee, "Enhanced FFT-based parametric algorithm for simultaneous multiple harmonics analysis," Proc. Inst. Elect. Eng., Gen. Transm. Distrib., vol. 148, pp. 209-214, May 2001.

[13] J. Z. Yang and C. W. Liu, "A precise calculation of power system frequency and phasor," IEEE Trans. Power Del., vol. 15, no. 2, pp. 494-499, Apr. 2000.

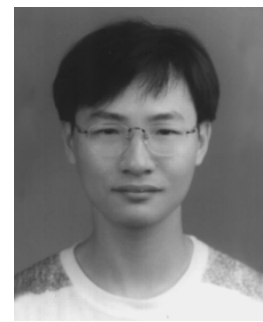

Jun-Zhe Yang (M'00) was born in Tainan, Taiwan, R.O.C., in 1971. He received the B.S. degree in electrical engineering from Tantung University, Taipei, Taiwan, in 1994 and the M.S. and Ph.D. degrees in electrical engineering from Taiwan University, Taipei, in 1997 and 2000, respectively.

Currently, he is an Assistant Professor of electrical engineering at I-Shou University, Kaoshiung, Taiwan. He was in compulsory military service for two years. His research areas are power quality and computer relaying.

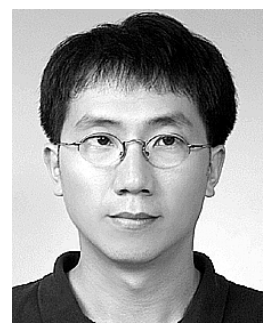

Chi-Shan Yu was born in Taipei, Taiwan, R.O.C., in 1966. He received the B.S. and M.S. degrees in electrical engineering from National Tsing Hua University, Hsinchu, Taiwan, in 1988 and 1990, respectively, and the $\mathrm{Ph} . \mathrm{D}$. degree in electrical engineering from National Taiwan University, Taiwan, in 2001.

Currently, he is an Assistant Professor of electrical engineering with National Defense University, Chung-Cheng Institute of Technology, Taoyuan, Taiwan. From 1991 to 2001, he was with Private Kuang-Wu Institute of Technology and Commerce, Taipei. His research areas are in computer relaying, power system transient stability controller design, and power electronics.

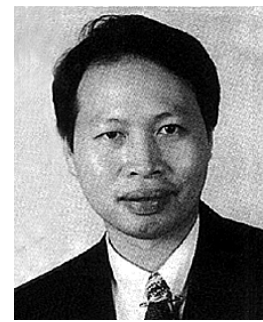

Chih-Wen Liu (S'93-M'96-SM'03) was born in Taiwan, R.O.C., in 1964. He received the B.S degree in electrical engineering from National Taiwan University, Taiwan, in 1987, and the M.S and $\mathrm{Ph} . \mathrm{D}$. degrees in electrical engineering from Cornell University, Ithaca, NY, in 1992 and 1994 respectively.

Currently, he is Professor of electrical engineering with National Taiwan University. His main research interests include application of computer technology to power system monitoring, operation, protection, and control. His other research interests include motor control and power electronics. He serves as a reviewer for IEEE TRANSACTIONS ON POWER SYSTEMS and IEEE TRANSACTIONS ON POWER DELIVERY. 